\title{
Modificación de la función renal y la presión arterial inducida por antiinfla- matorios no esteroideos de uso odon- tológico en terapia de corto plazo
}

Renal function and arterial pressure changes induced by odontological short term therapy nonsteroidal anti-inflammatory drugs

\section{Resumen}

El objetivo del presente estudio fue evaluar las posibles modificaciones en la presión arterial, peso corporal y depuración de creatinina en orina recolectada durante 24 horas, en sujetos jóvenes sanos, que han sido sometidos a terapia con dos antiinflamatorios no esteroideos (AINES); uno de ellos no selectivo de Ciclooxigenasa (Ibuprofeno) y un inhibidor selectivo de Ciclooxigenasa - 2 (Celecoxib), durante tres días en dosis estandarizadas, frente a un grupo control al que se le administró placebo, en un estudio doble ciego.

Los resultados indican que no existen diferencias estadísticamente significativas entre los tres grupos, para los parámetros clínicos y de laboratorio evaluados $(p<0,05)$.

Se concluye que la terapia de corto plazo con los AINES empleados, no producen modificaciones de impacto clínico en la presión arterial, peso corporal y depuración de creatinina en orina de 24 horas.

\begin{abstract}
The aim of the study was to evaluate the possible modifications in the arterial pressure, corporal weigh and creatinine cleareance in urine gathered during 24 hours, in healthy young people, under therapy with two non steroidal anti inflammatory drugs (NSAID's); one of them not selective to Ciclooxigenase (Ibuprofen) and a selective inhibitor of Ciclooxigenase - 2 (Celecoxib), during three days in standardized dose, in comparison with a control group that was administered placebo, in a double blind study.

The results indicate that there are not statistically significant differences among the three groups, for the clinical and laboratory evaluated parameters $(p<0,05)$.

We conclude that a short term therapy with the used NSAID's doesn't produce clinical impact modifications in the arterial pressure, corporal weigh and creatinine cleareance in urine gathered during 24 hours.
\end{abstract}

\section{Introducción}

Los antiinflamatorios no esteroideos (AINES) son fármacos prescritos a nivel mundial. Aproximadamente del 1 al $5 \%$ de pacientes bajo tratamiento con éstos fármacos experimentan algún tipo de alteración renal que requiere de la intervención del facultativo ${ }^{1}$. En nuestro país ocupan uno de los primeros lugares en la prescripción, ya sea por intermedio del prescriptor o por la inducción a su uso a través de propaganda radial y televisiva. La frecuencia de fallo renal agudo en pacientes que no ingieren AINES es de uno por cada 100,000 habitantes. Esta cifra se duplica o cuadriplica en los que sí reciben AINES, observándose en numerosos estudios que este evento está relacionado con la frecuencia, dosis, edad y estado fisiopatológico renal $^{2}$. Los objetivos de la presente investigación fueron evaluar las posibles modificaciones en la presión arterial, peso corporal y depuración de creatinina en orina recolectada durante 24 horas, en sujetos jóvenes que han sido sometidos a terapia con dos antiinflamatorios no esteroideos (AINES); uno de ellos no selectivo de Ciclooxigenasa (Ibuprofeno) y un inhibidor selectivo de Ciclooxigenasa - 2 (Celecoxib), durante tres días ${ }^{3}$ en dosis estandarizadas, frente a un grupo control al que se le administró placebo.

\section{Miguel Óscar Rodríguez Alfaro, Víctor Manuel Chumpitaz Cerrate ${ }^{1}$, Jonny Burga Sánchez', Roger López Bellido', Jorge Arturo Ramón Rosales ${ }^{1}$, Roberto Carlos Varas Hilario ${ }^{1}$, Juan Manuel Zegarra Cuya².}

Departamento Académico de Ciencias
Básicas
Bachiller en Odontología
1,2 Facultad Odontología. UNMSM

Correspondencia:

Mg. Miguel Rodríguez Alfaro Facultad Odontología, UNMSM

Av. Germán Amézaga s/n, Lima, 1 Perú. e-mail: miguelrodrigueza@hotmail.com

Palabras clave: Antiinflamatorios no esteroideos (AINES), depuración de creatinina, ciclooxigenasa.

Key words: Non-steroidal antiinflammatory drugs, creatinine cleareance, ciclooxigenase
La enzima ciclooxigenasa (COX) que interviene en la formación de prostaglandinas fisiológicas y pro inflamatorias se expresa en 2 isoformas: La COX - 1 y la COX - $2^{4}$. Las principales complicaciones renales se deben a la disminución de la síntesis de prostaglandinas fisiológicas. Las prostaglandinas más importantes para la adecuada función renal son la PGI2 y la PGE2. Estas mantienen una adecuada perfusión sanguínea renal y están mediadas en su formación por la enzima Cox - 1 y Cox - 2, las cuales son constitutivas en el riñón ${ }^{5,6,13,14}$. Estas prostaglandinas incrementan su expresión fisiológica con una dieta 
baja en sal, su inhibición produce alteraciones renales como la retención de sodio (con lo cual el paciente puede ganar kilos de peso en períodos cortos), edema periférico e incremento de la presión arterial ${ }^{7}$. Uno de los mecanismos de daño renal inducido por AINES es el relacionado al disparo de mecanismos apoptóticos, en relación a un incremento del estrés oxidativo en las nefronas posiblemente generado por neutrófilos activados y disfunción mitocondrial. 8,9

Los signos cardinales de esta nefrotoxicidad inducida por AINES son la elevación del nitrógeno, urea y creatinina en sangre, ganancia de volumen corporal y disminución del volumen urinario $^{1}$. En cuanto a la relación del daño con la duración de la terapia, en odontología la principal prescripción de AINES para manejar dolor e inflamación postoperatorios no sobrepasa las 48 a 72 horas de administración, vale decir terapias de corto plazo, lo que hace diferente su manejo en relación con otras especialidades médi$\operatorname{cas}^{10}$. El valor estimado normal para la depuración de creatinina en orina, fue obtenido a partir del uso del Método Cinético de Química Líquida (método de Jaffe modificado), estando su rango entre los 80 y $140 \mathrm{ml}$ / min, siendo éste un método exacto para la determinación del valor de depuración, que contrasta los resultados con la aplicación de la fórmula de Cockcroft-Gault, que da como valor indicativo de daño renal promedio las medidas que estén por debajo de $60 \mathrm{ml} / \mathrm{min}^{11}$.

Las prostaglandinas mantienen la función renal. La COX-1 se encuentra en el glomérulo y la arteriola aferente, mientras COX - 2 se localiza en los podocitos, mácula densa, porción gruesa del asa de Henle, y la arteriola aferente, manteniendo el flujo sanguíneo y el tono vascular normal ${ }^{12}$. Estas prostaglandinas derivan tanto de la COX-1 como de la COX - 2. Esto explicaría el daño renal producido por la ingesta de AINES no selectivos, además de predecir el daño que se produce con el uso clínico de los inhibidores selectivos de COX $-2^{13}$. El daño renal puede manifestarse a través del síndrome nefrótico. Las drogas que mayormente inducen nefrotoxicidad son los antibióticos, AINES, los medios de radiocontraste, antineoplásicos y drogas antirreumáticas ${ }^{14}$.

Los metabolitos de COX-2 están implicados en el mantenimiento del flujo de sangre renal, la mediación de descarga de renina, y la regulación de la excre- ción de sodio. La inhibición de COX-2 puede disminuir el volumen de orina temporalmente, disminuyendo la excreción de sodio en algunos pacientes e inducir una moderada elevación de la presión arterial. ${ }^{15}$

\section{Materiales y método}

El presente estudio fue del tipo experimental, clínico con un grupo control. Se seleccionó un total de 36 sujetos mayores de edad (alumnos del segundo y tercer año de estudios de la Facultad de Odontología de la UNMSM) que cumplieran con los criterios de inclusión especificados en el consentimiento informado que se les entregó previamente. Estos, en resumen, incluían principalmente que no debían estar recibiendo medicación alguna desde 15 días antes del inicio de la investigación ni tener antecedentes de enfermedades renales. Estos pacientes hombres y mujeres se asignaron de manera aleatoria a alguno de los tres grupos formados, los cuales fueron:

a. Grupo 1: (Placebo)

b. Grupo 2: Ibuprofeno $400 \mathrm{mg}$ tres veces al día.

c. Grupo 3: Celecoxib $200 \mathrm{mg}$ dos veces al día.

En todos los casos, la duración del tratamiento fue de tres días.

El primer día del estudio a todos los participantes se les tomó una muestra de sangre para determinar la concentración de creatinina en suero (pretratamiento) con la finalidad de hacer el cálculo de la depuración de creatinina en orina. Además recibieron las indicaciones necesarias para recolectar una muestra de orina durante 24 horas para determinar la depuración de creatinina (pretratamiento).

Al día siguiente de haber recolectado la muestra de orina, cada uno de los participantes inició la terapia con los fármacos correspondientes a su grupo. Asimismo cada uno de los días que duró el tratamiento acudieron al centro de investigación clínica para controlar su peso y presión arterial.

Luego de finalizado el tratamiento de tres días con las drogas indicadas, a cada uno de los pacientes se les volvió a tomar una muestra de sangre para evaluar la concentración de creatinina en suero (postratamiento) con la finalidad de hacer el cálculo de la depuración de creatinina en orina y se les dio instrucciones para la reco- lección de orina de 24 horas para la determinación de la depuración de creatinina (postratamiento).

\section{Resultados}

Los resultados del análisis de la variación de la presión arterial sistólica entre los grupos placebo, ibuprofeno y celecoxib, en cada tiempo de estudio no fue estadísticamente significativa con un $\mathrm{p}<0,05$. Con el análisis de Tukey se comprueba que no hubo variación significativa entre los tres grupos durante el tiempo de estudio. (Gráfico 1).

\section{Gráfico 1. Variación de la presión arterial sistólica}

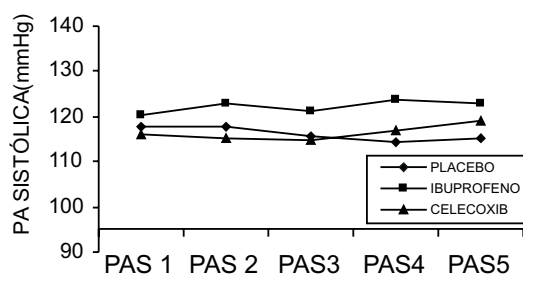

TIEMPO (DIAS)

Se observa una variación de la presión arterial sistólica durante el tiempo de estudio, sin embargo los valores promedio de cada grupo estuvieron dentro de los rangos normales.

Los resultados del análisis de la variación de la presión arterial diastólica entre los grupos placebo, ibuprofeno y celecoxib, en cada tiempo del estudio no fue estadísticamente significativa con un $p<0,05$. Con el análisis de Tukey se comprueba que no hubo variación significativa entre los tres grupos durante el tiempo de estudio (ver gráfico 2).

\section{Gráfico 2. Variación de la presión arterial diastólica}

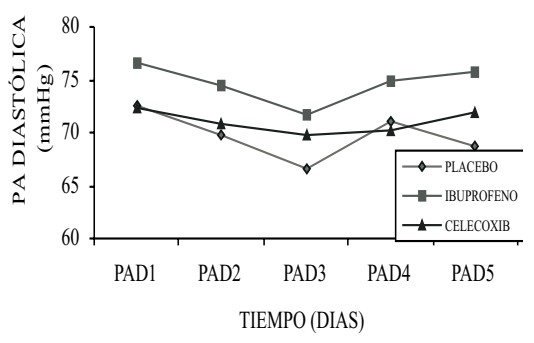

Se observa una variación de la presión arterial diastólica durante el tiempo de estudio, sin embargo los valores promedio de cada grupo estuvieron dentro de los rangos normales

El análisis de la variación de la depuración renal de creatinina entre los grupos placebo, ibuprofeno y celecoxib, en cada tiempo de estudio no fue estadísticamente significativa con 
un $\mathrm{p}<0,05$. Con el análisis de Tukey se comprueba que no hubo variación significativa entre los tres grupos (ver gráfico 3).

Los sujetos de estudio fueron pesados el día pre - tratamiento (peso 1), los tres días del tratamiento (pesos 2,3 y 4) y el día postratamiento (peso 5). Los resultados del análisis de la variación del peso corporal entre los grupos placebo, ibuprofeno y celecoxib, en cada tiempo de estudio no fue estadísticamente significativa con un $\mathrm{p}<0,05$. Con el análisis de Tukey se comprueba que no hubo variación significativa con un $\mathrm{p}<0,05$ (ver gráfico 4 ).

\section{Gráfico 3. Variación de la depura- ción de creatinina por AINES}

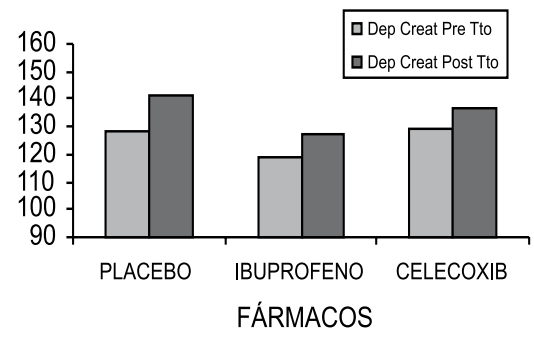

Se observa que los valores promedios de depuración de creatinina postratamiento fueron mayores que los valores pre -tratamiento, sin embargo los promedios se encuentran dentro de los rangos normales

\section{Gráfico $\mathrm{N}^{\circ} 4$. Variación del peso corporal producido por AINES}

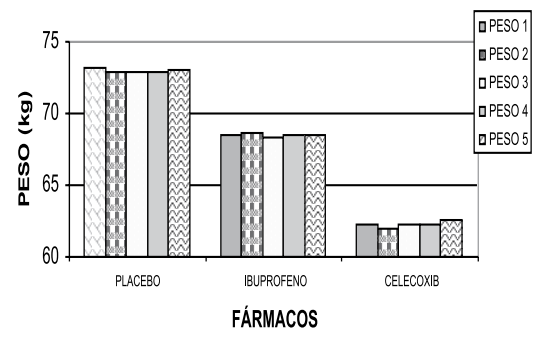

No hubo variación del peso corporal dentro de cada grupo. El grupo placebo presento valores promedio de peso corporal mayor al resto de los grupos del estudio, siendo el grupo de celecoxib el que presentó menor promedio de peso corporal.

Se observaron algunas reacciones adversas medicamentosas, siendo el tracto gastrointestinal el principal afectado, ya que el riesgo de incremento de úlceras gastrointestinales y sangrado digestivo alto se incrementa hasta en cuatro veces con el empleo de AINES ${ }^{22}$, observándose este efecto con el uso de ibuprofeno en dos sujetos. Este riesgo se incrementa adicionalmente con la edad avanzada. Otras reacciones adversas medicamentosas reporta- das fueron somnolencia, edema de miembros inferiores y rash dérmico, presentándose cada una de ellos en un paciente.

\section{Discusión}

Los antiinflamatorios no esteroideos (AINES) son ampliamente usados para el tratamiento del dolor agudo y crónico, y condiciones inflamatorias. Sin embargo, tal vez estos agentes estén asociados con diferentes efectos adversos, incluyendo efectos sobre la función renal. Los AINES actúan inhibiendo ambas isoformas de ciclooxigenasa COX-1 y COX-2 lo cual en adición a los efectos benéficos tal vez contribuya a reacciones adversas por disminución de prostaglandinas protectoras. El desarrollo de inhibidores de COX-2 fue basado sobre la hipótesis que por inhibición de COX-2 estos agentes podrían ser eficaces como los AINES tradicionales y tener incidencia menor de efectos gastrointestinales, sin embargo los datos de estudios han demostrado que los inhibidores de COX-2 tienen los mismos efectos renales que los AINES tradicionales. ${ }^{5}$

Según el presente estudio los AINES empleados no presentan efectos adversos a nivel renal, esto se evidencia debido a que ninguno de los fármacos, tanto el ibuprofeno como el celecoxib disminuyeron la depuración de creatinina. Esto puede explicarse porque en el estudio los límites de edad estuvieron comprendidos de 17 a 36 años, perteneciendo la mayoría de 20 a 25 años, lo que en cierta medida puede hacer que los efectos adversos renales de los AINES podrían verse enmascarados, pero deben realizarse más estudios para determinar que grupo atareo presenta mayor predisposición a los efectos adversos de los AINES.

Hay evidencias inequívocas de que en el riñón están presentes tanto COX-1 y COX-2 en formas constitutivas e inducible, lo que sugiere que los inhibidores selectivos Cox-2 tengan los mismos efectos en las prostaglandinas renales que los AINES tradicionales. ${ }^{16,17}$ Las prostaglandinas (PGs) que son las más importantes del riñón son PGE2 y PGI2 o prostaciclina. La PGE2 disminuye la reabsorción del sodio en la rama ascendente gruesa del asa de Henle y la PGI2 estimula la liberación del renina, que concomitantemente aumenta la aldosterona. La aldosterona aumenta la reabsorción del sodio y la secreción del potasio en el nefrón distal. La prostaciclina es también un potente vasodilatador que mantiene la velocidad de filtración glomerular (GFR) y el flujo renal de la sangre de pacientes con disminución de volumen. ${ }^{18,19}$ En individuos sanos, el papel vasodilatador de PGI2 no es operativo y tiene poca importancia en la hemodinámica renal. Entendiendo los efectos fisiológicos de las prostaglandinas renales y de su función las consecuencias de inhibirlas pueden ser previsibles. La inhibición de PGE2 causará la retención de sodio, que puede manifestarse como aumento del peso, edema periférico, o raramente, insuficiencia cardiaca congestiva ${ }^{20}$. La presión arterial puede también aumentar debido a la retención del sodio. Esto ocurre más comúnmente en los pacientes hipertensos que están en tratamiento de con antihipertensivos. En estos pacientes, los meta-análisis han demostrado que la magnitud de efecto sobre la presión arterial es un aumento de aproximadamente 5 $\mathrm{mm} \mathrm{Hg}$ o menos en varias semanas. Además, la inhibición de PGI2 puede causar hiperkalemia o en pacientes en riesgo, falla renal aguda. ${ }^{21}$

En el presente estudio no se encontró un aumento estadísticamente significativo de la presión arterial tanto en los pacientes que ingirieron ibuprofeno y celecoxib frente al control esto puede deberse tal vez a que el aumento de la presión arterial necesita de un tiempo más prolongado mayor a 3 días de ingesta de AINES.

El peso corporal no tuvo un aumento considerable, lo que indica que los AINES tradicionales y selectivos en terapia corta de 3 días no afectan en mayor cuantía la producción de la PG E2, la cual al no causar la retención de sodio no producirá edema y por lo tanto no se evidenciará el aumento de peso.

Según los datos obtenidos del estudio, la terapia corta con Ibuprofeno y celecoxib están libres de efectos adversos sugiriendo esto, que tal vez para que se evidencien los efectos adversos renales por la ingesta de AINES se necesite un tiempo mayor a los 3 días de tratamiento.

Los datos sugieren que en terapia corta de 3 días, que es lo que se suele prescribir en Odontología, sean seguros al no modifican la función renal y que debe ser entendida como una práctica segura cuando se emplee por un tiempo no mayor de 3 días.

Al término del estudio se llegó a las siguientes conclusiones:

1. La terapia de corto plazo con los AINES empleados no produce modificaciones de impacto clínico en la presión arterial. 
2. La terapia de corto plazo con los AINES empleados no produce modificaciones de impacto clínico en el peso corporal.

3. La terapia de corto plazo con los AINES empleados no produce modificaciones de impacto clínico en depuración de creatinina en orina de 24 horas.

\section{Agradecimiento:}

Al CSI de la UNMSM por el apoyo financiero para la ejecución del proyecto.

\section{Referencias Bibliográficas:}

1. Whelton A. Nefrotoxicity of non streoidea anti-inflamatory drugs: physiologic foundations and clinical implications. Am J Med 1999; 106:13-24.

2. Hernández - Díaz, S., García L. Epidemiologic asessment of the safety of conventional non streoideal anti - inflamatory drugs. Am J Med 2001; 110: 20-27.

3. Rodríguez M. Antiinflamatorios no esteroideos y su uso en odontología: una vieja costumbre. Odont Sanmarqu 1999; 1: 40-43.

4. Mitchell, Warner T Ciclooxigenase 2: Pharmacology, physiology, biochemistry and relevance of NSAIDs therapy. British Journal of Pharmacology 1999; 128: 1121- 1132.

5. Brater C. Effects of non streoideal ant - inflamatory drugs on renal function focus on ciclooxigenase - 2 selective inhibition. Am J Med 1999; 107: 65-71.

6. Cicconetti A, Bartoli A, Ripari F, Ripari A. Cox 2 selective inhibitors: A literatura review of analgesic efficacy and safery in oral - maxillofacial surgery. Oral Surg Oral Med Oral Pathol 2004; 97: 139-46.

7. Harris R. Ciclooxigenase-2 inhibition and renal physiology. Am J Cardiol 2002; 89: 10-17.

8. Hickey E, Raje R, Reid V, Gross M, Ray D. Diclofenac induced in vivo nefrotoxicity may involve a oxidative stress-mediated massive genomic DNA fragmentation and apoptotic cell death. Free Radical Biology \& Medicine. 2001; 31: 139-152.

9. Basivireddy J, Jacob M, Pulimood AB, Balasubramanian KA. Indomethacininduced renal damage: role of the oxygen free radicals. Biochemical Pharmacology 2004; 67: 587-599.

10. Haas D. Adverse drug interactions in dental practice: interactions associated with analgesics. JADA 1999; 130: 397406.

11. Stürmer T, Erb A, MPharm, Keller F, Günther K, Brenner $H$, Determinants of Impaired Renal Function with Use of Nonsteroidal Anti-inflammatory Drugs: The Importance of Half-life and Other MedicationsAm J Med. 2001; 111: 521-527.

12. DeMaria A, Weir M. Coxibs-Beyond the GI Tract: Renal and Cardiovascular Issues. J Pain Symptom Manage. 2003; 25:41-49.

13. Parente L, Perreti M. Advances in the pathophysiology of constitutive and inducible ciclooxigenases: two enzymes in the spotlight. Biochemical pharmacology 2003. 65: 153 - 159.

14. Ohno I.Drug induced nephrotic syndrome.Nippon Rinsho.2004Oct;62( 10):1919-24

15. Cheng HF, Harris RC. Cyclooxygenases, the kidney, and hypertension.
Hypertension. 2004 Mar;43(3):525-30. Epub 2004 Jan 19.

16. DeMaria A, Weir MR. Coxibs - Beyond the GI Tract: Renal and Cardiovascular Issues. J Pain Symptom Manage 2003;25: 41-49.

17. Parente L, Perretti M. Advances in the pathophysiology of constitutive and inducible cyclooxygenases: two enzymes in the spotlight. Biochemical Pharmacology 2003; 65: 153-159.

18. Clive DM, Stoff JS. Renal syndromes associated with non-steroidal antiinflammatory drugs. N Eng J Med 1984; 310: 563-572.

19. Harris RC. Cyclooxygenase-2 in the kidney. J Am Soc Nephrol 2000;11:23872394.

20. Juhlin T, Bjorkman S, Gunnarsson B, Fyge A, Roth B, Hoglund P. Acute administration of diclofenac, but possibly not long term low dose aspirin, causes detrimental renal effects in heart failure patients treated with ACEinhibitors. The European Journal of Heart Failure 2004; 6: 909- 916.

21. Murray MD, Black PK, Kuzmik DD, et al. Acute and chronic effects of nonsteroidal anti-inflammatory drugs on glomerular filtration rate in elderly patients. Am J Med Sci 1995;310:188197.

22. Griffin M, Scheiman J, Prospects for Changing the Burden of Nonsteroidal

Anti-Inflammatory Drug Toxicity. 2001 The American J. of Medicine. 110 (1A):33-37

Recibido : 26-04-2007

Aceptado para publicación: 25-05-2007

\section{PROYECTOS DE INVESTIGACIÓN APROBADOS - 2007}

- Título: Efecto antimicótico del croton draconoides en estomatitis subplaca de pacientes edentulos portadores de prótesis. Fase I

Responsable: Gálvez Calla, Luis

- Título: Elementos ambientales de riesgos que determinan la aparición de caries dental en indivíduos de 6 a 36 meses de edad, que acuden a la Clínica Integral del Niño de la Facultad de Odontología de la UNMSM durante el año 2007

Responsable: Castañeda Mosto, María

- Título: Prevalencia y factores que intervienen en las enfermedades asociadas a portadores de fisura labio máxilo-palatina en hospitales de Lima y Callao Responsable: Guillén Borda, Celso 